viii

\title{
On Nets of Polygons Occurring in Nature.
}

\author{
By W. H. M`Crea.
}

1. The section of certain vegetable cell structures takes normally the form of a net of hexagons. When a departure from this form occurs it is observed to be such that three edges still meet at each vertex of the net and the average number of sides per polygonal mesh is still six. After noting this fact, Graustein ${ }^{1}$ shows in a recent paper that under these conditions the average number of sides is necessarily precisely six. He then generalises the geometrical result. The present note derives the same results by an entirely different process. The arguments are of a physical character and do not pretend to be mathematically rigorous. But it seems worth while to put them forward as indicating just why the effect should occur in Nature.

2. The simplest form of the original theorem is:-

Suppose that from $a$ net of $L$ hexagons any interior sets be removed and replaced by any set of polygons subject only to the condition that three, and only three, edges meet at a vertex. Then if the modified system contain in all $P$ polygons and $S$ sides we have $S=6 P$, precisely.

By an edge we denote a linear segment, so that each interior edge is a side of two polygons.

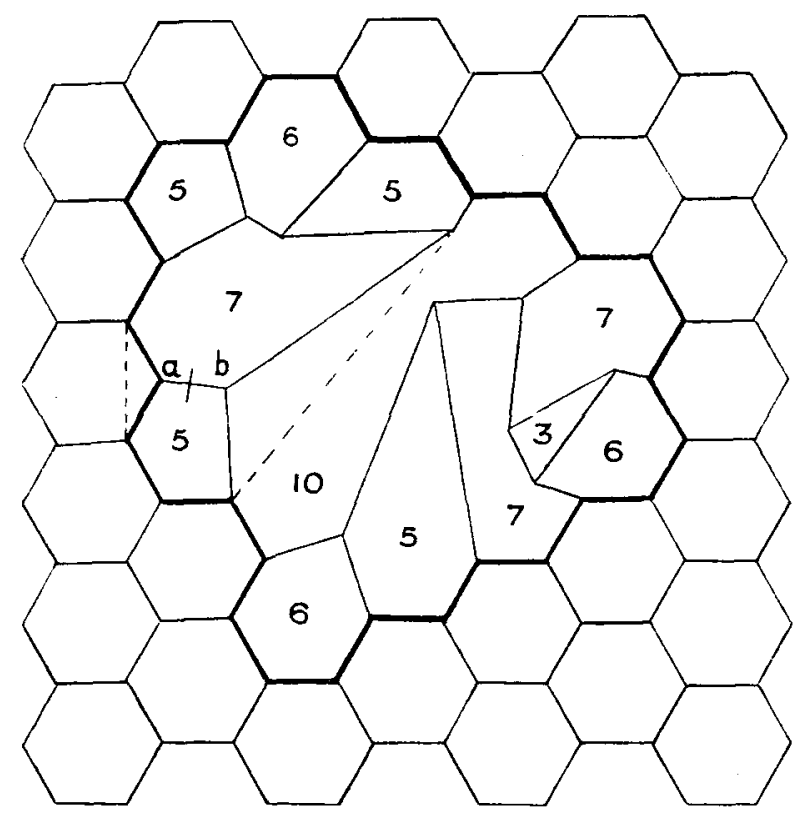

Fig. 1

I W. C. Graustein, Annals of Mathematics (2), 32 (1931), p. 149. 
We start with the modified net. This will consist of segments belonging to the original hexagons, and "new" edges introduced in forming the new polygons. Suppose now the whole thing be replaced by an exactly similar net made of pieces of elastic knotted together at the vertices, the whole being attached to a rigid frame round the outer boundary. Let it take up a position of equilibrium. This may alter the shapes of the meshes but in no way alters their number, or the numbers of their edges. Now suppose any interior edge be cut. The two edges connected to it at each end will at once straighten out. (Fig. $1 c f . a b$ ). Therefore the total number of edges is reduced by three, i.e., one cut and two pairs straightening into two single segments. The number of sides is consequently reduced by six, and at the same time the number of cells is obviously reduced by one. By a repetition of this process, $N$ times" (say), all the " new" edges can be removed. This reduces the number of cells by $N$ and the number of sides by $6 N$.

The original hexagons may now be replaced. This can be done by the repetition, $M$ times (say), of the process of tying in a fresh piece of elastic between points on two sides of the gap to be repaired. Each process is the exact converse of a cutting process. So $M$ cells and $6 M$ sides are added. The repaired net has $L$ hexagonal cells, and so $6 L$ sides.

We have therefore on counting up the number of cells removed and added

$$
P-N+M=L
$$

and the number of sides removed and added

$$
S-6 N+6 M=6 L \text {. }
$$

So we have at once

$$
S=6 P
$$

Thus the modified net has an average of precisely six sides per cell.

3. This method of treating the problem gives insight into the reason for the occurrence of the phenomenon in Nature. It shows how the collapse of cell walls can never alter the average number of sides per cell.

4. It is clear that the above arguments depend on the fact that three edges per vertex are enough, and just enough, for the equilibrium of

${ }^{2} N$ is in general less than the number of "new" edges on account of the straightening out phenomenon. 
the elastic net with every side in tension. Hence the method cannot be applied immediately to nets with more than three edges per vertex.
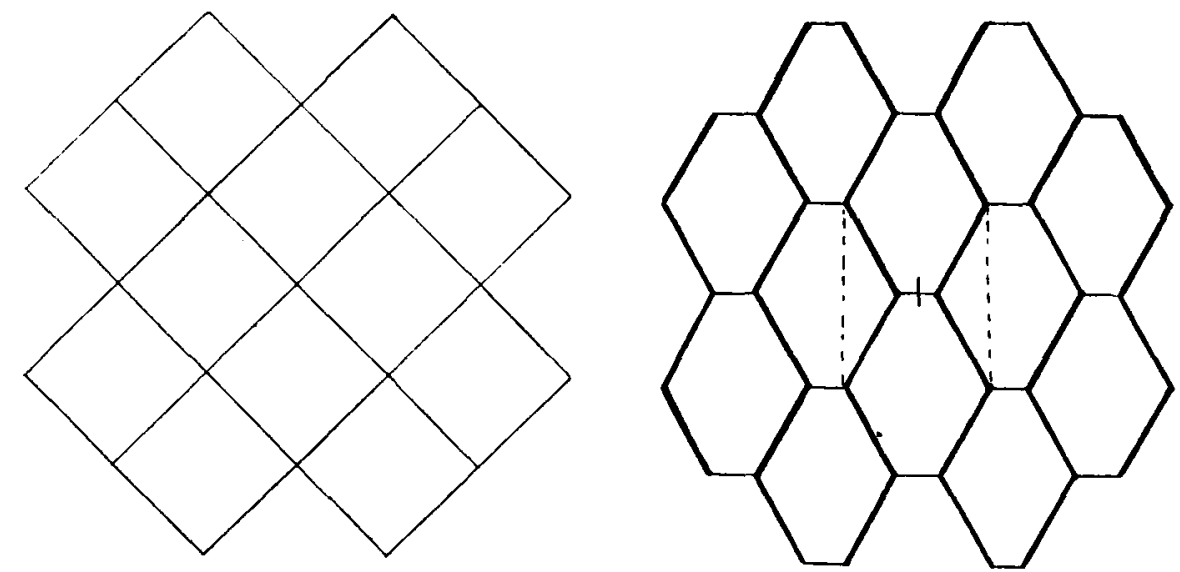

Fig. 2

A net with four edges per vertex can nevertheless be made into one with three per vertex by inserting an extra edge at each vertex as shown in Fig. 2. If we do this, and then apply our cutting process to any number $N$ of these inserted edges, and finally let those remaining shrink back to zero length, we shall reduce the number of cells by $N$ and the number of original sides by $4 N$. For the removal of an added side will not count in the final result. We may not cut any of the original edges, since this would violate the condition of four edges per vertex in the final figure. By completing the argument as in $\$ 2$ we get the theorem: If from a net of rectangles any interior sets be removed and replaced by polygons so that four, and only four, edges meet at a vertex, then the average number of sides per polygon is always precisely four.

5. Finally a net of triangles with six edges per vertex can be replaced by a net with three per vertex by inserting a hexagon at each vertex. (Fig. 3). If in this we cut any of the original edges and then let the added edges shrink to zero length we get a system without six edges per vertex. If we cut some of the inserted edges and let the remaining ones tend to zero, then either the final figure is unaltered (cutting $l$, Fig. 3), or it is without six edges per vertex (cutting $m, n$ ). 
Hence the only (interior) modification admitted by such a net of triangles is a deformation which does not alter the number of cells or vertices.
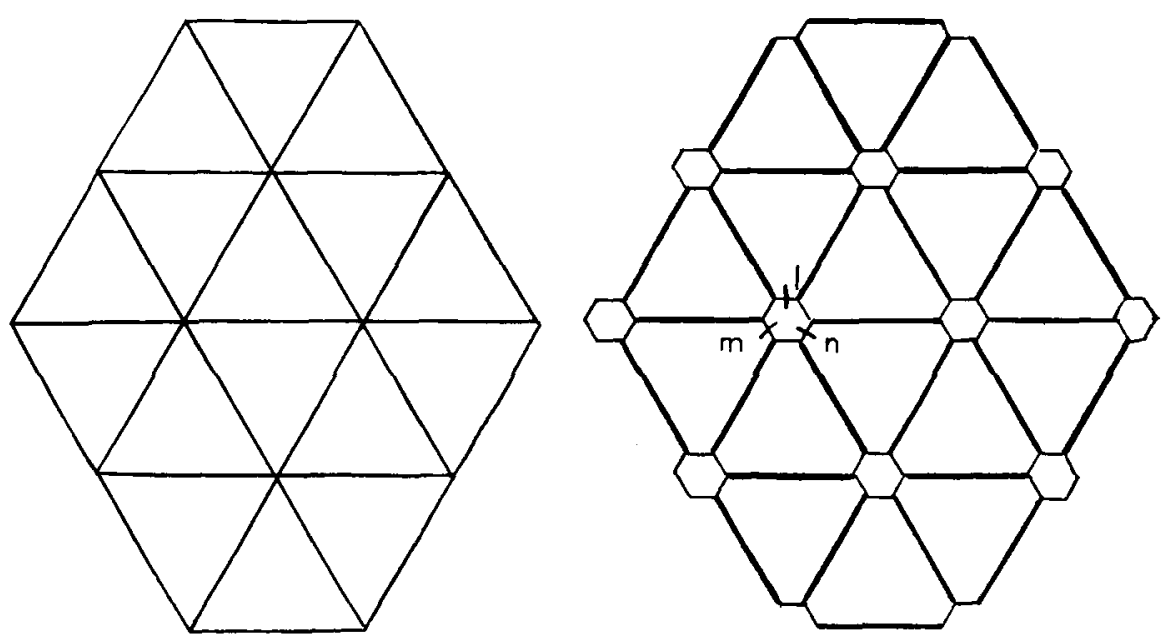

Fig. 3

These theorems are all proved geometrically by Graustein.

Incidentally the last one shows that: If in any system with three edges per vertex we know that every edge is either a side of a hexagon, or terminates at the vertex of a hexagon, it necessarily follows that every cell is a hexagon.

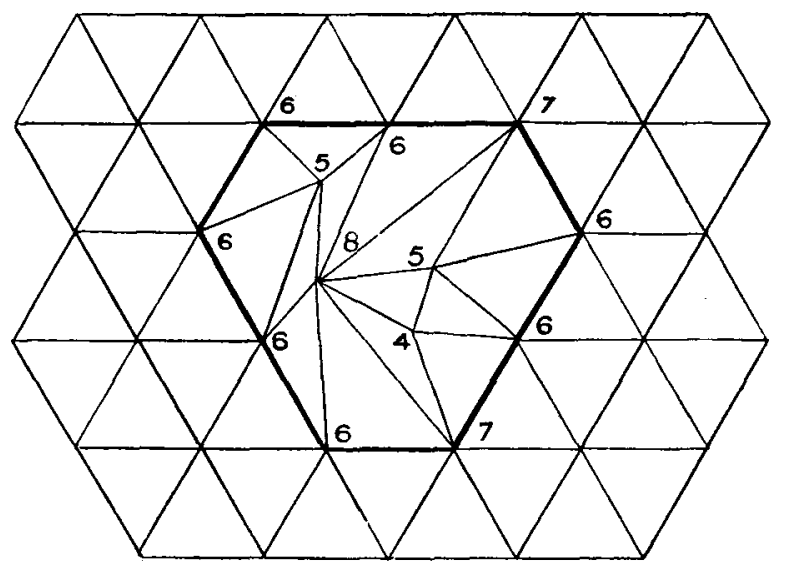

Fig. 4 
xii

6. I am much indebted to Professor H. W. Turnbull for pointing out to me the existence of a set of reciprocal theorems which may be obtained from the "force diagrams" for the original nets. They are:-

(i) If from a net of triangles with six edges per vertex, any interior set be removed and the vacated space be filled with triangles in any manner, then the average number of edges per vertex is precisely six. (Fig. 4)

(ii) If from a net of quadrilaterals with four edges per vertex any interior set be removed, and the vacated space be filled with quadrilaterals in any manner, then the average number of edges per vertex remains precisely four.

(iii) The only (interior) modification in a net of hexagons, which replaces them by hexagons, is a mere deformation.

\section{A Note on Stirling's Theorem}

By J. R. Wilton.

Let $\Gamma(1+x)=\sqrt{ }(2 \pi x) x^{x} e^{-x} \phi(x) ;$

then

$$
\lim _{x \rightarrow \infty} \phi(x)=1 \text {. }
$$

This result is Stirling's theorem. A simple proof is given in $\$ 1.87$ of Titchmarsh's Theory of Functions (Oxford Univ. Press, 1932).

Rather more than Stirling's theorem can be proved by a method which assumes nothing but the definition of the $\Gamma$-function, and $\Gamma\left(\frac{1}{2}\right)=\sqrt{ } \pi$, from which it follows that

$$
\begin{aligned}
& \text { if } x>0, \int_{0}^{\infty} e^{-\frac{1}{2} x \tau^{2}} d \tau=\sqrt{\left(\frac{\pi}{2 x}\right),} \\
& \text { and } \quad \int_{0}^{\infty} \tau^{2} e^{-\frac{1}{2} x \tau^{2}} d \tau=\frac{\sqrt{ } \pi}{x \sqrt{ }(2 x)} .
\end{aligned}
$$

The method is not new. It was given, in essentials, in lectures by the late R. A. Herman, at the beginning of the century; and it may be much older. 